\title{
LA CONSTRUCCIÓN DE UN MODELO PEDAGÓGICO DEL EMPODERAMIENTO JUVENIL: ESPACIOS, MOMENTOS Y PROCESOS
}

\author{
THE CONSTRUCTION OF A PEDAGOGICAL MODEL \\ OF YOUTH EMPOWERMENT: SPACES, MOMENTS AND PROCESSES
}
A CRIAÇÃO DE UM MODELO PEDAGÓGICO DA CAPACITAÇÃO JUVENIL: ESPAÇOS, MOMENTOS E PROCESSOS

\author{
Pere SOLER MASO*, Jaume TRILLA BERNET**, Manel JIMÉNEZ-MORALES*** \\ \& Xavier ÚCAR MARTÍNEZ**** \\ *Universitat de Girona, ${ }^{* *}$ Universitat de Barcelona, ${ }^{* * *}$ Universitat Pompeu Fabra, \\ **** Universitat Autònoma de Barcelona
}

PALABRAS CLAVES: empoderamiento jóvenes modelo pedagógico educación pedagogía social
Fecha de recepción del artículo: 24.11.2017 Fecha de revisión del artículo: 6.111.2017 Fecha de aceptación final: 4.v.2017

\begin{tabular}{|c|c|}
\hline $\begin{array}{l}\text { PALABRAS CLAVES: } \\
\text { empoderamiento } \\
\text { jóvenes } \\
\text { modelo pedagógico } \\
\text { educación } \\
\text { pedagogía social }\end{array}$ & $\begin{array}{l}\text { RESUMEN: El presente artículo es fruto de un proyecto de investigación (EDU2O13-42979-R) } \\
\text { en el que se profundiza desde diferentes perspectivas en el empoderamiento juvenil. En la } \\
\text { aportación que aquí presentamos partimos de la pregunta inicial de dónde, cuándo y cómo } \\
\text { tiene lugar el empoderamiento juvenil. Se pretende construir un modelo educativo que pue- } \\
\text { da servir de orientación para ordenar y estudiar estos espacios, momentos y procesos. Se } \\
\text { parte de un extenso trabajo previo de análisis documental en el que se formulaba una pri- } \\
\text { mera conceptualización del empoderamiento juvenil a partir del análisis de } 297 \text { referencias } \\
\text { bibliográficas posteriores al año } 2000 \text { que abordaban desde una perspectiva socioeducativa } \\
\text { el empoderamiento juvenil. En la presente aportación partimos de este trabajo realizado y en } \\
\text { esta ocasión la metodología utilizada ha consistido en el análisis reflexivo de dichas fuentes } \\
\text { documentales y la posterior sistematización de los datos obtenidos con el propósito de cons- } \\
\text { truir una propuesta teórica razonada y convenientemente argumentada. } \\
\text { El artículo fundamenta una definición propia de empoderamiento juvenil centrada en } \\
\text { trabajos anteriores del equipo de investigación y en el enfoque de capacidades de M. Nuss- } \\
\text { baum. Se articula un patrón general, en forma de modelo pedagógico, con el propósito de } \\
\text { presentar un referente con el que poder describir, explicar e interpretar el empoderamiento } \\
\text { de los jóvenes, así como proyectar y orientar intervenciones para optimizarlo. Se concluye } \\
\text { con unas reflexiones finales sobre ciertas formas posibles pero indeseables de entender o } \\
\text { instrumentalizar los procesos de empoderamiento. }\end{array}$ \\
\hline
\end{tabular}

CONTACTAR CON LOS AUTORES: Pere Soler Maso. Universitat de Girona. Departament de Pedagogia. Plaça de Sant Domènec, 9. 17004 Girona (Spain). pere.soler@udg.edu. 


\begin{tabular}{|c|c|}
\hline $\begin{array}{l}\text { KEY WORDS: } \\
\text { empowerment } \\
\text { young people } \\
\text { pedagogical model } \\
\text { education } \\
\text { social pedagogy }\end{array}$ & $\begin{array}{l}\text { ABSTRACT: The present paper is the result of a research project (EDU2O13-42979-R) which } \\
\text { investigated youth empowerment from different perspectives. The initial question was where, } \\
\text { when and how youth empowerment takes place. The aim was to build an educational model } \\
\text { that could serve as guidance for the organization and study of these spaces, moments and } \\
\text { processes. The starting point was a previous extensive documentary analysis that formulated } \\
\text { an initial conceptualization of youth empowerment. It was based on the review of } 297 \text { biblio- } \\
\text { graphical references from the year } 2000 \text { onwards that addressed youth empowerment from } \\
\text { a socio-educational perspective. The methodology consisted in the reflective analysis of the } \\
\text { abovementioned documentary sources and the subsequent systematization of the data ob- } \\
\text { tained, in order to build a reasoned and well-argued theoretical proposal. } \\
\text { This paper provides its own definition of youth empowerment by focusing on previous } \\
\text { studies carried out by the research team and on M. Nussbaum's capabilities approach. A } \\
\text { general pattern was constructed in the form of an pedagogical model intended to offer a } \\
\text { benchmark to describe, explain and interpret youth empowerment, as well as to plan and } \\
\text { guide interventions intended to optimize it. We conclude with some final reflections on some } \\
\text { possible-but undesirable-ways to understand and exploit these empowerment processes. }\end{array}$ \\
\hline $\begin{array}{l}\text { PALAVRAS-CHAVE: } \\
\text { capacitação } \\
\text { jovens } \\
\text { modelo pedagógico } \\
\text { educação } \\
\text { pedagogia social }\end{array}$ & $\begin{array}{l}\text { RESUMO: O presente artigo é fruto de um projeto de investigação (EDU2O13-42979-R) no } \\
\text { qual se aprofunda a capacitação juvenil de várias perspetivas. No contributo que aqui apre- } \\
\text { sentamos, partimos da pergunta inicial de onde, quando e como acontece a capacitação ju- } \\
\text { venil. Pretende-se criar um modelo educativo que possa servir de orientação para ordenar } \\
\text { e estudar estes espaços, momentos e processos. Parte-se de um extenso trabalho prévio } \\
\text { de análise documental no qual se formulou uma primeira conceptualização da capacitação } \\
\text { juvenil a partir da análise de } 297 \text { referências bibliográficas posteriores ao ano } 2000 \text { que } \\
\text { abordavam a capacitação juvenil de uma perspetiva socioeducativa. No presente contributo, } \\
\text { partimos deste trabalho realizado e, nesta ocasião, a metodologia utilizada consistiu na aná- } \\
\text { lise reflexiva das ditas fontes documentais e na posterior sistematização dos dados obtidos } \\
\text { com o propósito de elaborar uma proposta teórica bem fundamentada e convenientemente } \\
\text { argumentada. } \\
\text { O artigo fundamenta uma definição própria de capacitação juvenil centrada em trabalhos } \\
\text { anteriores da equipa de investigação e na abordagem de competências de M. Nussbaum. } \\
\text { Articula-se um padrão geral, em forma de modelo pedagógico, com o propósito de apresen- } \\
\text { tar um referente com o poder de descrever, explicar e interpretar a capacitação dos jovens, } \\
\text { assim como projetar e orientar intervenções para o otimizar. Conclui-se com umas reflexões } \\
\text { finais sobre certas formas possíveis, mas indesejáveis, de entender ou instrumentalizar os } \\
\text { processos de capacitação. }\end{array}$ \\
\hline
\end{tabular}

\section{Introducción}

La bibliografía existente sobre empoderamiento es abundante. Sin embargo, la que se refiere específicamente al empoderamiento juvenil no lo es tanto. Falta, por ejemplo, un marco de referencia o un modelo general que sirva para cartografiar los procesos juveniles de empoderamiento. Esta es la propuesta que presentamos en las páginas siguientes: un modelo con el que poder describir, explicar e interpretar el empoderamiento de los jóvenes, así como proyectar y orientar intervenciones para optimizarlo.

Con este modelo se pretende conocer y ofrecer ordenadamente algunas respuestas a la pregunta siguiente: ¿dónde, cuándo y cómo se produce el empoderamiento? Esto significa determinar cuáles son los espacios, los momentos y los procesos empoderadores más significativos. Se trata de tres perspectivas de análisis distintas, pero complementarias y de ninguna manera excluyentes entre sí. Son enfoques complementarios que necesariamente deberán confluir si se pretende ofrecer una interpretación holística y coherente del empoderamiento juvenil. No hay ningún proceso que se dé al margen de un contexto determinado de espacio y tiempo; ni ningún tiempo que pueda estudiarse al margen del espacio en que transcurre, etc. Ahora bien, reconocida esta complementariedad de los tres elementos del modelo, habrá que hacer énfasis también en la necesidad de definir, con la máxima precisión posible y diferencialmente, los contenidos propios y específicos de lo que llamamos espacios, momentos y procesos.

Este trabajo es parte de un proyecto de investigación más amplio', del que en este mismo número de la revista se ofrecen otros resultados. El antecedente más directo de este artículo es otro muy reciente de los mismos autores (Úcar, Jiménez-Morales, Soler y Trilla, 2016), en el que realizábamos un estudio bibliográfico en torno al empoderamiento y formulábamos una conceptualización del mismo. El presente artículo -de carácter más reflexivo y sistematizador- no hubiera sido posible sin el conocimiento de las fuentes y aportaciones previas que nos proporcionó el estudio mencionado². En esta aportación, la 
metodología utilizada ha consistido en el análisis reflexivo de las citadas fuentes documentales y la posterior sistematización de los datos obtenidos con el propósito de construir una propuesta teórica razonada y convenientemente argumentada.

Además de la presentación del modelo, el artículo incluye otros dos apartados. Uno previo sobre el concepto de "empoderamiento" que, como es bien sabido, es un término que está todavía lejos de resultar unívoco. Esa es la razón para estipular el concepto de empoderamiento antes de entrar en el modelo. El artículo se cierra con unas breves reflexiones, suscitadas a partir de un par de citas literarias, sobre ciertas formas posibles pero indeseables de entender o instrumentalizar los procesos de empoderamiento.

\section{Una propuesta conceptual sobre el empoderamiento}

El concepto de empoderamiento que vamos a manejar parte de un trabajo bibliográfico previo que pretendía, justamente, contribuir a la conceptualización del término (Úcar et al., 2016) a partir, entre otros, de autores de referencia como Rappaport (1981, 1987), Freire (1987) y Zimmerman (2000).

Bauman define a la persona empoderada como aquella que es "capaz de elegir y de actuar conforme a lo elegido y eso, a su vez, implica la capacidad de influir en el conjunto de acciones alternativas disponibles y en los escenarios sociales en los que se eligen y materializan esas opciones" (Bauman, 2010, p. 270). Partimos pues de que el núcleo más esencial de la idea de empoderamiento se refiere a dos capacidades diferentes pero sucesivas: la capacidad de decidir; y la capacidad de actuar de forma consecuente con lo decidido. Ciertamente, el empoderamiento supone algo más que poder decidir: es además poder llevar a cabo aquello que se ha decidido. Que uno esté capacitado para poder decidir ciertamente ya es un logro; pero si no lo está también para poder desarrollar sus decisiones y actuar de acuerdo con ellas, aquel logro lo que mayormente producirá será frustración.

Ahora bien, hay otra pregunta a la que es necesario dar respuesta: ¿Sobre qué tipos de asuntos ha de ir siendo capaz de decidir y actuar la persona en proceso de empoderamiento? $Y$ aquí la respuesta también ha de ser doble. Alguien aumenta su grado de empoderamiento en la medida en que mejora su capacidad para:

- tomar decisiones y actuar de forma consecuente sobre aquello que afecta a su propia vida;

- y poder participar en la toma de decisiones e intervenir de forma compartida y responsable en lo que afecta a la colectividad de la que forma parte y al medio en el que vive.

En el lenguaje ordinario solemos diferenciar bastante bien entre aquellas decisiones que tomamos en función de lo que, directa y prioritariamente, afectará a nuestra vida propia; y las decisiones que tomamos en función de lo que, directa y prioritariamente, creemos que afectará a los demás o a otras instancias (a nuestra familia, comunidad, nación, humanidad, medio ambiente, etc.). Es cierto que aquello que decidimos y hacemos para los demás también afecta a nuestra vida propia; y viceversa, que las decisiones sobre nuestra propia vida van a afectar también, de un modo u otro, a los demás, al entorno, etc. Y es verdad: todo está relacionado con todo, todo afecta a todo. Pero del hecho cierto de que lo que hacemos para nosotros mismos repercute en los demás, y lo que hacemos para los demás repercute en uno mismo, no se sigue que debamos darle el mismo trato. Conviene por tanto distinguir entre lo que sirve para poder controlar autónomamente la propia vida, y lo que sirve para poder participar en asuntos públicos (sociales, cívicos, políticos...). Creemos que el concepto de empoderamiento debe acoger conjuntamente ambas dimensiones. Haciendo énfasis sólo en la capacidad para decidir sobre la vida propia se corre el riesgo de caer en un concepto solipsista o individualista y meritocrático de empoderamiento; un concepto coherente con ciertos planteamientos del actual neoliberalismo pujante. Desde esta perspectiva el empoderamiento estaría muy relacionado con otro concepto de moda: emprendeduría. Y el modelo ideal de persona empoderada y emprendedora sería el del self-made-man.

Para precisar un poco más el concepto de empoderamiento que vamos a manejar hay que clarificar otra distinción importante. Para poder realmente decidir (o poder participar en la toma de decisiones) y actuar de forma consecuente, han de darse como mínimo dos condiciones; ambas igualmente necesarias. Si no se dan las dos, la decisión y, sobre todo, la actuación consecuente, no serán factibles.

La primera condición es que el sujeto disponga de determinadas capacidades que podemos denominar internas o personales o psicológicas (conocimientos, actitudes, aptitudes, valores, habilidades...) $)^{3}$. Las capacidades personales a las que nos referimos serán, en cada caso, las necesarias o convenientes para poder tomar las decisiones de que se trate y llevarlas a cabo. Esta primera condición es de naturaleza específicamente educativa, dado que tales capacidades se desarrollan por medio de la educación. 
La segunda condición es que el entorno posibilite que el sujeto pueda decidir y llevar a cabo su decisión. Aquí se incluirían los condicionamientos políticos, económicos, legales, materiales... citados antes, pero también aspectos como normas y presiones sociales, estereotipos, modas, etc.

Para poder decidir y llevar a cabo la decisión de que se trate han de darse tanto las condiciones internas como las externas. Para decidir que la lectura forme parte de mi ocio es imprescindible saber leer (condición interna) pero también tener libros a mi alcance (condición externa). Para poder votar en unas elecciones la legalidad política vigente ha de establecerlo (externa), pero para votar con responsabilidad y conocimiento de causa es necesario también disponer de la información precisa sobre las distintas opciones (interna). De hecho, el empoderamiento real es siempre el resultado de una relación dialéctica entre ambas condiciones ${ }^{4}$ :

Aunque ambas condiciones sean imprescindibles en relación a un empoderamiento real, para aplicar el modelo que presentaremos después y que nos permita comprender mejor cuándo, dónde y cómo se empoderan las personas, es conveniente distinguirlas analíticamente. Ello, porque no sería nada extraño que ciertos espacios, momentos o procesos sirvan más para lo uno que para lo otro. Seguramente podremos comprobar que determinados espacios o procesos actúan más eficazmente facilitando formación empoderadora (condición interna), y otros como facilitadores de recursos materiales, económicos, legales, etc. (condiciones externas). $Y$ también podremos comprobar que hay espacios que hacen ambas cosas a la vez (espacios máximamente empoderadores), y otros que no hacen ninguna de ellas o incluso se dedican a poner trabas (espacios nulamente empoderadores o desempoderadores).

A partir de todo lo anterior y para resumir, proponemos la siguiente caracterización del concepto que nos ocupa:

Empoderamiento es el proceso que incrementa las posibilidades de que una persona pueda decidir y actuar de forma consecuente sobre todo aquello que afecta a su propia vida, participar en la toma de decisiones e intervenir de forma compartida y responsable en lo que afecta a la colectividad de la que forma parte. Esto requiere dos condiciones: que la persona vaya adquiriendo y desarrollando una serie de capacidades personales (conocimientos, actitudes, aptitudes, destrezas...) y que el medio le facilite ejercer efectivamente tales capacidades 5 .

En la fig. 1 representamos esquemáticamente nuestra idea de empoderamiento poniendo de relieve la acción determinante de la educación y el medio.

Figura 1. El concepto de empoderamiento

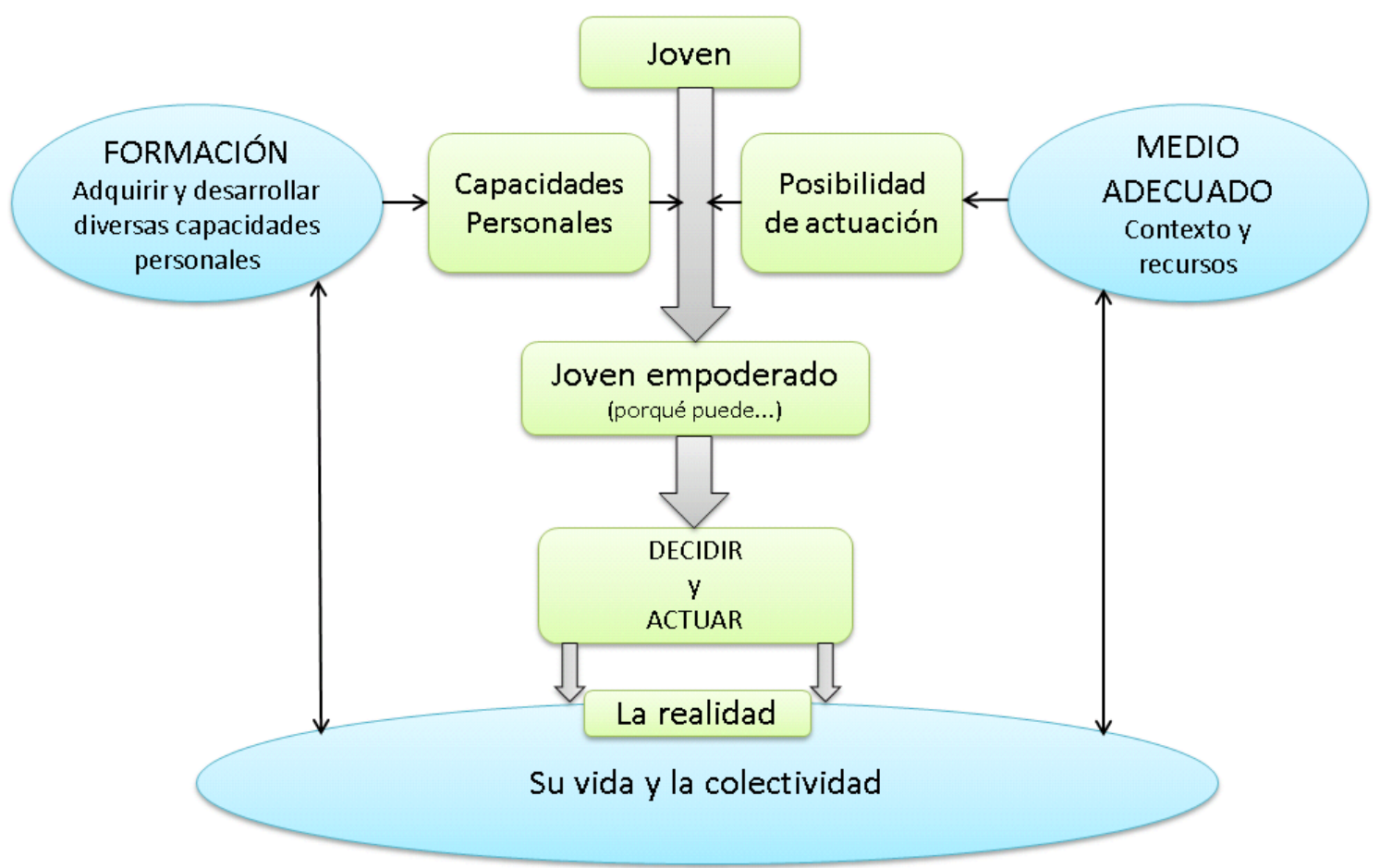


Consideramos cualquier proceso de empoderamiento como un proceso educativo (Shirazi, 2011), aunque no únicamente. Sin educación no puede haber empoderamiento, pero no basta con ella, no es suficiente. Como hemos dicho, para que se pueda decidir y actuar de forma consecuente con esta decisión hace falta también un contexto determinado. Somerville (1998) ya hacía notar la necesidad de diferentes elementos y procesos para alcanzar el empoderamiento, además de la educación. Apuntaba asimismo la legislación, la movilización de recursos y la transferencia de poder. En este sentido cabe considerar que el medio, el contexto social y político, su estructura y servicios, son también un potente agente educativo. En algunos casos este medio puede ser un buen aliado para promover el empoderamiento, pero en otras ocasiones la dificultad será justamente encontrar la forma de evitar, contrarrestar o eludir el efecto de este medio limitador, empobrecedor, acomodador o alienador.

\section{El dónde, el cuándo y el cómo del empoderamiento juvenil}

\subsection{Los espacios}

En esta categoría situamos todo aquello que da respuesta a la pregunta sobre dónde se produce o manifiesta el empoderamiento de los jóvenes. Seguramente referirse a espacios es algo parecido a hablar de "contextos", "ámbitos", "entornos" e "instituciones". En este punto se recogen todo este conjunto de denominaciones, que utilizan los autores al referirse al empoderamiento.

En distintos trabajos se han propuesto clasificaciones, tipologías y criterios para identificar y ordenar los espacios de empoderamiento más significativos (Luttrel et al., 2009; Shaw et al., 2014; Boluijt \& de Graaf, 2010; Gaventa, 2006; Masa, 2009). Por nuestra parte presentamos primero un repertorio amplio de lugares, real o potencialmente, relacionados con el empoderamiento juvenil, para después proponer algunos criterios y distinciones que faciliten analizarlos adecuadamente. Lo que sigue no pretende ser una nueva propuesta taxonómica, sino un simple muestrario para evidenciar la gran diversidad de espacios significativos para el empoderamiento juvenil a los que diversos autores se han referido.

- Instituciones educativas formales: institutos de enseñanza secundaria, universidades, centros de formación profesional... (Peterson et al., 2011; Ozer \& Schotland, 2011; Pearrow \& Pollack, 2009; Messias et al., 2005; San Saturnino \& Gaicoechea, 2013).
- Instituciones educativas no formales y de educación social: centros juveniles, instituciones y grupos de educación en el tiempo libre, instituciones y servicios de educación social... (Lawrencejacobson, 2006; Lakin \& Mahoney, 2006; Wright, 2010; Wong, 2008).

- Asociaciones políticas, ciudadanas, religiosas...: partidos políticos, movimientos sociales, organizaciones no gubernamentales, confesiones religiosas... (Claret, 2013; Messias et al., 2005).

- Instituciones y equipamientos culturales, artísticos, deportivos, sanitarios...: museos, bibliotecas, clubs deportivos, hospitales y centros de salud... (Cargo et al., 2003; Kronenberg, 2007).

- Espacios ciudadanos públicos: plazas, parques, la calle, centros comerciales y recreativos... (Nolas, 2014; Somerville, 1998; Pato, 2014; Trilla et al., 2011).

- El ámbito familiar: los distintos tipos de familia y también las instituciones de suplencia o substitución de la misma. (Kaplan, Skolnik \& Turnbull, 2009; Roth \& Brooks-Gunn, 2003).

- El ámbito laboral (Richez et al., 2012; Cloete \& Auriacombe, 2013).

- El espacio virtual, las TIC y los medios de comunicación. (Carlsson et al., 2008; Fortunati, 2014; Qiu, 2008; Wang, 2006; Subirats \& Parés, 2014).

\subsubsection{Los espacios como práctica y promoción del empoderamiento}

Los distintos espacios indicados pueden analizarse y valorarse desde una doble perspectiva: en qué medida los sujetos se encuentran empoderados respecto a cada uno de ellos; y en qué medida cada espacio puede generar en los sujetos algún tipo de empoderamiento transferible a otros espacios. Se trata de dos dimensiones diferentes -aunque complementarias- de la relación entre espacio y empoderamiento.

La primera dimensión es la que considera a los espacios como ámbitos en los que se manifiesta, expresa y pone en práctica el empoderamiento. Como es obvio, el grado de empoderamiento de cada sujeto depende, en buena medida, del espacio concreto de que se trate. La segunda dimensión en la que los espacios resultan significativos respecto al empoderamiento es la que entiende que tales espacios son empoderadores. Es decir, ámbitos que inciden -positiva o negativamente- en el empoderamiento de los sujetos. Y ello, no sólo dentro del espacio concreto del que se trate, sino también en relación al resto de espacios. La capacidad empoderadora de un espacio 
determinado ha de medirse no sólo por el empoderamiento que genera en los sujetos dentro del propio espacio sino, también, por cómo dicho empoderamiento puede transferirse a otros espacios. Esta capacidad de transferencia se debe fundamentalmente a la intrínseca dimensión educativa del empoderamiento a la que nos referíamos antes.

En el repertorio de espacios presentado cabría identificar algunos sobre los que, por su propia naturaleza y sentido, debe recaer una mayor responsabilidad empoderadora. Pero eso entendiendo que el empoderamiento, como cualquier otro aprendizaje o capacidad, solo se adquiere o desarrolla mediante la práctica. La familia o los centros educativos ejercerán como agentes de un empoderamiento transferible a otros espacios si -y sólo si- en su propio seno permiten realmente a los jóvenes ejercer el poder que les corresponda. Una familia en la que predominen las relaciones autoritarias -y/o paternalistas- difícilmente será espacio para un empoderamiento transferible a otros espacios.

\subsubsection{Espacios específicamente juveniles y espacios intergeneracionales}

Entre los distintos espacios presentados los hay que son específica o preponderantemente juveniles y otros que son intergeneracionales. Entre los primeros cabría distinguir dos subtipos. Uno sería el constituido por aquellos espacios que son juveniles porque institucionalmente está establecido que sus miembros o usuarios pertenezcan a esta franja de edad: un centro juvenil, un centro de enseñanza secundaria, un punto de información juvenil, un centro de acogida para jóvenes... A estos lugares los llamaremos espacios juveniles institucionalizados. Pero también existen otros espacios preponderante -o incluso casi exclusivamente- frecuentados por jóvenes, sin que ello haya sido formalmente establecido: espacios que de facto seleccionan a sus usuarios por la clase de actividad que en ellos tiene lugar o por haberse convertido en lugares de encuentro y relación muy mayoritariamente juvenil (skateparks, determinadas zonas urbanas y locales comerciales y de ocio...). A estos los denominaremos espacios juveniles informales. $\mathrm{Y}$ por último tendríamos otros espacios -también muy significativos para el empoderamiento de los jóvenes- que son, por su propia naturaleza, intergeneracionales: la familia, el ámbito laboral, muchas instituciones y equipamientos culturales, etc.

En general, los espacios juveniles informales pueden ser considerados como ámbitos generados para que en ellos los jóvenes puedan gozar de unos márgenes de autonomía -y de poder propio- superiores al que pueden disponer en los otros ámbitos. En estos espacios informales de socialización horizontal los jóvenes, apartados de la supervisión y censura adulta, pueden expresar su idiosincrasia más genuina. Es ahí donde los jóvenes ponen en práctica su juventud y, por tanto, donde verdaderamente pueden ser jóvenes. Como contrapartida, una visión crítica de tales espacios aduciría que en ellos lo que se manifiesta y reproduce son mayormente algunos de los estereotipos más tópicos e indeseables que recaen sobre la juventud (consumismo, frivolidad, carpe diem irresponsable...). Su paradigma sería entonces el botellón, un ejemplo claro de las contradicciones que comporta el ocio como espacio de aprendizaje juvenil (Comas, 2001).

Por su parte, los espacios juveniles institucionalizados, desde una perspectiva positiva, son los más favorablemente preparados para promover un empoderamiento transferible. De hecho, a la gran mayoría de tales espacios se les reconoce una función explícita y prioritariamente educadora (centros de enseñanza, instituciones de educación social y de animación sociocultural...). Y es esa función formativa la que convierte a tales instituciones en espacios privilegiadamente empoderadores, en el sentido de adquisición de competencias transferibles a otros espacios. Hay que apuntar, sin embargo, que puede haber educación de muchas clases. Entre ellas, educación empoderadora y educación desempoderadora; y esa última, aunque indeseable, no es menos educación que la primera. Hay ciertamente procedimientos educativos para formar a personas dóciles, serviles, sumisas, conformistas y dependientes. Incluso hay quien diría que en ciertas instituciones educativas lo que realmente se aprende es a necesitar ser enseñado, a necesitar que siempre haya quien te prescriba lo que hay que aprender y cómo hay que aprenderlo. Para que las instituciones educativas que trabajan con jóvenes formen en competencias empoderadoras transferibles a otros espacios, deben empezar por convertirse ellas mismas en espacios donde los jóvenes puedan ejercer realmente su parte de poder.

Nuestras sociedades son cada vez más plurales en muchos aspectos (raza, origen, cultura, ideología y religión...), y quizá precisamente por eso tienden cada vez más a configurarse a base de crear espacios específicos y diferentes para unos y otros: unos para niños, otros para jóvenes y otros para viejos; barrios para ricos y barrios para pobres, para inmigrantes y para autóctonos... Pero ello no debería nunca degenerar en ghettos que, más que por elección, acaban funcionando por exclusión. Recluir a los jóvenes en reductos 
o espacios propios -por más que pudieran parecer paraísos artificiales- no es la mejor manera de empoderarles, sino una forma de paternalismo muy eficaz para excluirlos. Esa es la razón por la que los espacios intergeneracionales deberían considerarse como lo normal antes que como la excepción.

Los espacios intergeneracionales deberían ser la mejor referencia del empoderamiento de los jóvenes. Si hay que empoderar a los jóvenes no es para que ellos puedan participar en las decisiones que sólo afectan a los propios jóvenes, sino para que puedan participar en las decisiones que afectan al conjunto de la comunidad de la que forman parte. Es en los espacios intergeneracionales donde los jóvenes aprenden a participar en lo que les afecta a ellos pero también en lo que afecta a todos.

\subsection{Los momentos del empoderamiento juvenil}

En este apartado se trata la segunda cuestión del modelo: el cuándo del empoderamiento. ¿El empoderamiento se produce de forma diferente según etapas o períodos? ¿Hay unos momentos que son más propicios que otros para empoderarse?

\subsubsection{El empoderamiento y las etapas del ciclo vital}

En primer lugar, cabe pensar que los momentos del empoderamiento se refieren a los diversos periodos de la vida (infancia, juventud, adultez, vejez). Parece obvio que esta variable cronológica es importante en el momento de estudiar cómo se produce el empoderamiento y cómo hay que intervenir para promoverlo. Si el empoderamiento de los jóvenes posee algunas características propias, sólo es posible elucidarlas a base de contrastar esta etapa del ciclo vital con las demás, y especialmente con las inmediatamente anterior (infancia) y posterior (adultez).

Asimismo, cabría también la posibilidad de considerar subetapas en el desarrollo psicosocial de los jóvenes, pues entre uno de 18 años y otro de 28 las diferencias pueden ser bien significativas. Comparativamente, esta distancia de sólo diez años resulta mucho más relevante en las edades indicadas que, pongamos por caso, entre una persona adulta de 38 y otra de 48 años.

\subsubsection{El empoderamiento y los momentos de la vida cotidiana}

La pregunta sobre el cuándo del empoderamiento juvenil podría asignar a la palabra «momentos» un significado distinto al que le hemos dado en el apartado anterior. Ahora podríamos entender que los momentos a los que nos referimos son determinados segmentos temporales de la vida cotidiana. Puede tener mucho sentido investigar y reflexionar sobre cuestiones como «el empoderamiento en los períodos vacacionales», "la vida nocturna y el empoderamiento de los jóvenes», "la hora de volver a casa de los adolescentes y los conflictos familiares que ello conlleva», etc., etc. (Masa, 2009; Wright, 2010).

El transcurrir de la vida cotidiana es, sin duda, un tema importante en relación al empoderamiento. Empoderarse también significa aprender a controlar el tiempo propio. Esto supone, al menos, dos cosas. En primer lugar, ser consciente del uso real que hacemos de nuestro tiempo cotidiano: ver en qué actividades lo empleamos y valorar el grado de satisfacción que ellas nos proporcionan. $Y$ una vez concienciado el uso real que se hace del tiempo cotidiano, habrá que ver la forma de optimizar su uso de acuerdo con objetivos y criterios autodefinidos. Hacerse consciente del uso actual del tiempo propio y aprender a administrarlo de forma autónoma, responsable y satisfactoria serían pues adquisiciones empoderadoras relevantes.

\subsubsection{El empoderamiento y los acontecimientos críticos (critical events)}

También son especialmente significativos para el empoderamiento aquellos acontecimientos que, pudiendo ser de naturaleza muy variada, comparten el hecho de ser vividos con una especial intensidad: el primer amor o el primer fracaso sentimental, la desaparición de un ser querido, el momento de emanciparse y el abandono del hogar familiar, quedarse sin trabajo,... Ya se ve que unos serían positivos y otros negativos. Pero incluso estos últimos son momentos que pueden generar un mayor autoconocimiento, descubriéndole al joven facetas ocultas y reacciones y capacidades insospechadas. Experiencias que a veces nos hacen conscientes de nuestros límites, pero que en ocasiones nos hacen también descubrir que hay límites que parecían infranqueables y que no lo son tanto, porque hemos decidido afrontarlos y hemos sabido y podido superarlos. Por eso decimos que a veces estos momentos críticos pueden resultar empoderadores. Además algunos de ellos, por el hecho de devenir memorables, adquieren un rendimiento empoderador añadido: empoderaron en el momento en que fueron vividos y siguen haciéndolo cada vez que se rememoran. 


\subsubsection{El empoderamiento y determinados momentos o periodos sociales y colectivos}

Hasta aquí hemos estado tratando del tiempo personal de los jóvenes: de las etapas de su vida y de algunos de sus momentos, sean cotidianos o excepcionales. Pero también podría hablarse de periodos y momentos colectivos en tanto que factores de empoderamiento; momentos sociales que pueden resultar muy significativos para el empoderamiento de aquellos jóvenes que tuvieron la ocasión de vivirlos colectivamente. Por ejemplo, los hechos del mayo parisino de 1968 o el movimiento del $15 \mathrm{M}$ de 2011, por citar dos casos particularmente emblemáticos. Emblemáticos porque tuvieron como protagonistas principales a los jóvenes y también por el hecho de que ambos se identifican y han pasado a la historia con denominaciones que remiten al momento en que tuvieron lugar. Son numerosos los estudios sobre ambos acontecimientos ${ }^{6}$, y en ellos abundan testimonios de primera mano que ilustran de manera fehaciente procesos de empoderamiento vividos colectivamente por sus jóvenes participantes. Como éste de una participante en las movidas del $15 \mathrm{M}$ : «A título personal he aprendido mucho acerca de la democracia. Está siendo una lección que no se me va a olvidar en la vida. Y, a título colectivo, hemos aprendido a relacionarnos entre nosotros, a intentar actuar democráticamente, tomar las decisiones democráticamente de manera asamblearia y a relacionarnos con otros grupos». (Ana, trabajadora del sector sanitario, miembro del colectivo Indignados formado por miembros de su sector.), (AA.VV., 2011: 53).

Está claro que tampoco puede ser lo mismo empoderarse en el marco de una crisis económica, que hacerlo en épocas de desarrollo y bonanza. Ni la situación ni las necesidades de los jóvenes son las mismas en un contexto y en el otro; $y$ por tanto, ni los objetivos ni las prioridades ni las estrategias a aplicar en las políticas de empoderamiento tampoco pueden ser iguales.

\subsection{Los procesos de empoderamiento juvenil}

En este punto abordamos cómo se genera empoderamiento. Nuestro propósito es hacer una aproximación a aquellos métodos, sistemas, técnicas, procedimientos, programas y actividades que promueven, impulsan o facilitan el empoderamiento de los jóvenes. Proponemos un aproximación a estos procesos a partir de tres niveles: macro, meso y micro, según el alcance y dimensión de cada proceso.

\subsubsection{Los procesos empoderadores a nivel macro. El empoderamiento juvenil a través de las políticas públicas y de juventud}

¿Cuál debería ser el papel de las políticas públicas de juventud para favorecer el empoderamiento de los jóvenes? ¿Cómo actuar desde la administración sin caer en una excesiva tutela que limite la capacidad de decisión y actuación por parte de los jóvenes? Vamos a proponer algunas pistas que a nuestro modo de ver pueden servir de orientación y pauta en el abordaje del empoderamiento juvenil desde las políticas públicas.

a) La perspectiva educadora como elemento central de las políticas de juventud

La formación, capacitación y educación amplia y generosa es el primer principio esencial de cualquier proceso de empoderamiento. La educación y alfabetización política es imprescindible y determinante para posibilitar una decisión libre y consciente (Freire, 1997; 1997b). Este objetivo implica considerar la dimensión educadora en los distintos programas (salud, ocupación, vivienda, ocio, cultura, etc.) y no únicamente desde la educación reglada y ser consciente que el entorno o contexto de vida de los jóvenes es también un potente agente educativo.

b) Hacer posible el ejercicio real de los derechos reconocidos

La participación actual de los jóvenes en las estructuras de poder político, económico o social es insuficiente. Los espacios de toma de decisiones y de poder real han ido reduciéndose y alejándose de la ciudadanía y más aún de los jóvenes, a pesar de la evolución de los sistemas democráticos actuales. Los jóvenes han de recibir un mensaje de confianza y estímulo que los reconozca, los valore y motive para la acción y participación. Se trata de conseguir que tengan el poder real de influir, que lo sientan $y$, sobre todo, que lo puedan ejercer con garantías.

Ante este escenario hay propuestas a favor de la implicación de la ciudadanía en las decisiones de los gobiernos (Cornwall, 2008). Se reclama un estado más democrático en el que la participación sea entendida como un derecho básico a partir del cual se reclaman otros derechos y no sólo como un favor de los gobiernos, ni como un privilegio de los ciudadanos.

Si la participación de la ciudadanía en general es en muchos casos deficitaria, ¿Cómo podemos implicar a los jóvenes sin el ejemplo de los adultos? B. Checkoway (2011), 
Christens \& Peterson (2012) y Cornwall (2008) creen que las instituciones gubernamentales y no gubernamentales deberían intentar incrementar la implicación y representación de los jóvenes en su trabajo y en la mejora social y del sistema político.

c) Favorecer la autonomía e independencia de los jóvenes. El respeto al principio de subsidiariedad

La autonomía y la independencia han de ser consubstanciales al empoderamiento juvenil porque justamente se trata de que los jóvenes estén en disposición de decidir y actuar libremente y puedan ejercer la necesaria y sana colaboración, fiscalización y crítica tanto a la acción gubernamental, como también a la acción comunitaria. En este mismo sentido se manifiestan Richez, Labadie \& De Linares (2012) cuando afirman que la política de empoderamiento requiere fomentar la autonomía de los jóvenes, fortalecer su lugar en la sociedad y desarrollar la lógica democrática.

Esta forma de entender el diseño e implementación de las políticas públicas, y de manera especial las de juventud, ha de llevarse a cabo con respeto al principio de subsidiariedad. El objetivo es dar el máximo protagonismo a los jóvenes y reducir la acción directa por parte de la administración y de los adultos, reservándola para cuando se requiera de manera justificada.

\subsubsection{Los procesos empoderadores a nivel meso. El empoderamiento juvenil a través de grupos e instituciones}

Nos situamos ahora en un nivel intermedio entre las políticas (planificación y acción estratégica) y la acción directa sobre los grupos y personas (planificación y acción operativa). Correspondería a los procesos desarrollados mayoritariamente a través de los grupos e instituciones encargados de traducir las líneas políticas en programas y proyectos.

a) Programas abiertos, estimuladores de la creatividad y la libertad

Se requiere de procesos que permitan un margen de libertad, protagonismo y creatividad a los jóvenes. Sin esta libertad, el sujeto no puede experimentar y asumir el riesgo necesario que supone la toma de decisiones y su ejecución a través de la acción. Las instituciones y grupos que quieran promover el empoderamiento juvenil deberán poner los medios y herramientas necesarias para favorecer este clima e invitar al ensayo, la creación y la acción.

Según Kronenberg (2007), una atmósfera empoderadora debe permitir explorar, no categorizar los hechos artísticos como buenos o malos, permitir un nivel de errores a los jóvenes artistas para que crezcan, y valorar la imaginación de cada uno. Podríamos extrapolar la misma afirmación al resto de ámbitos y prácticas más allá de la esfera artística. Cuando los jóvenes sienten que son parte de un equipo, tienen responsabilidades y tienen una ambiente confortable en el que conseguir sus objetivos, su autoestima aumenta. Salusky et al. (2014) proponen además que los objetivos de los programas sean explícitos y que sean los jóvenes quienes piensen cómo lograrlos. Creen que no debe haber pautas a priori y que se favorece el empoderamiento cuando se considera a los jóvenes responsables, cuando hay expectativas respecto a ellos, cuando se les brinda apoyo y cuando hay trabajo en equipo con perseverancia.

b) Programas que contribuyan a la toma de conciencia

El empoderamiento juvenil requiere también una toma de conciencia personal y grupal/ comunitaria, según sea el contexto social e institucional. Ricaurte et al. (2013) proponen cuatro etapas que ejemplifican bien lo que puede requerir este proceso: a) conocer el pasado, b) comprender el presente, c) proyectar el futuro $y, d$ ) pasar a la acción y materializar la proyección. Para materializar estos pasos existen métodos y técnicas apropiadas. Se trata de propuestas que invitan a la reflexión, al descubrimiento, a la crítica, al debate abierto y al contraste de opiniones; todo lo necesario para configurar opinión propia y tomar conciencia sobre cuestiones esenciales. Siguiendo a Freire (1997) si las personas no son críticamente conscientes de las estructuras y procesos visibles e invisibles que constituyen las prácticas y las instituciones sociales, ni de su rol personal y acciones dentro de estas instituciones y prácticas, no queda lugar para el empoderamiento.

c) Técnicas específicas para el empoderamiento En este nivel pretendemos mencionar algunas técnicas concretas que se han identificado de forma explícita como un recurso reconocido en los procesos de empoderamiento. No entramos en las aportaciones de pedagogos célebres ni en propuestas pedagógicas reconocidas. En ellas también se podrían detectar propuestas concretas facilitadoras del empoderamiento.

Existen dos aportaciones muy concretas que en los últimos años se han configurado y definido propiamente como técnicas para el empoderamiento. Nos referimos al 
Photovoice y al Reflect-Acción. Wang (2006) presenta el Photovoice como una metodología para empoderar, que debe contener los siguientes pasos: (a) Seleccionar el público objetivo al que irán dirigidas las imágenes, historias o recomendaciones (políticos, periodistas, etc.); (b) Seleccionar el grupo de participantes y hacer un primer taller para explicar el proyecto y las nociones básicas de fotografía social; (c) Decidir conjuntamente la temática sobre la que se quiere incidir o proponer el tema específico del proyecto; (d) Proporcionar cámaras a los jóvenes y tiempo para hacer las fotos; (e) Abrir el diálogo sobre el significado de las fotografías a través de un debate estructurado 7 , planificar un formato para compartir las fotografías y las historias con el público elegido como destinatario (políticas, periodistas, comunidad, etc.).

Reflect-Acción ${ }^{8}$ (2009) consiste en una propuesta innovadora para la alfabetización y el cambio social que fusiona las teorías de Paulo Freire con la metodología del Diagnóstico Rural Participativo. Proporciona espacios democráticos donde reunirse y debatir para grupos de personas. Los participantes eligen libremente los temas de acuerdo a sus propias prioridades y con el apoyo de un facilitador. Pretende ayudar a las personas a luchar por sus derechos, a desafiar la injusticia y a cambiar su posición dentro de la sociedad. Para ello propone trabajar con la gente y no para la gente. Se concibe como un proceso político en el que las múltiples dimensiones del poder y de la estratificación social son siempre el centro de la reflexión y las acciones están encaminadas a cambiar las relaciones de poder injustas. Reflect-Acción se basa en una enorme gama de métodos participativos que buscan minimizar la dependencia y facilitar las condiciones para crear poder popular y empoderamiento.

Más allá del Photovoice y del Reflect-Acción hay diversas experiencias que presentan otros métodos y estrategias para el empoderamiento de grupos y comunidades. Por citar algunas: El Community-based service-learning (CBLS) como un método idóneo para el empoderamiento a través de programas comunitarios y propuestas pedagógicas comprometidas con la comunidad; Las $4 D$ (discover, dream, design and destiny), un método también de concienciación y superación; El mapeo comunitario a través del cual se construye la visión de la comunidad desde la misma comunidad. Mención especial merece el video participativo, puesto que ha sido una de las técnicas utilizadas por este equipo de investigación (Salvadó, Jiménez-Morales \& Sourdis, 2017). A través de la cámara, la filmación y la narración de historias se experimentan claros procesos de empoderamiento en la medida que los jóvenes toman conciencia de su realidad y adquieren un papel protagonista de su desarrollo.

\subsubsection{Los procesos empoderadores a nivel micro. Formas de actuar que promueven y facilitan el empoderamiento en las relaciones personales}

En este tercer grupo clasificamos aquellas pautas que tienen que ver con las actitudes, aptitudes, formas de actuar en las relaciones personales y en la acción cara a cara con jóvenes.

a) El establecimiento de una relación basada en la confianza

En las relaciones con los jóvenes la confianza en sus capacidades es un gran estímulo para la motivación y la superación personal. Cargo et al. (2003) explican la importancia de un ambiente propicio para que los jóvenes puedan responsabilizarse y ejercer de esta forma la transferencia de poder a la juventud entendida como la responsabilidad de expresarse, de tomar decisiones y de pasar a la acción.

b) Aprender haciendo, actuando

El fortalecimiento de las actitudes, aptitudes y competencias óptimas para el empoderamiento implica aprender haciendo (learning by doing) junto con otros. En este sentido se encuentran diversidad de ejemplos y experiencias con jóvenes, en las que la acción ejercida en primera persona es el eje central de la relación. Al fin y al cabo, el empoderamiento no se aprende, sino que se va consiguiendo a través de las acciones y formas de afrontar la vida. Desde esta consideración Shaw et al. (2014) proponen que muchas de las actividades de compromiso cívico sean consideradas óptimas para el empoderamiento juvenil: Actividades comunitarias y de voluntariado, ayuda mutua, etc.

c) La presencia / ausencia de los adultos Los procesos demasiado dirigidos, acompañados o pautados pueden limitar las posibilidades de empoderamiento. La acción ideal sería aquella en la que se consiga el justo equilibrio entre la guía y la libertad de acción. Messias et al. (2005) y Salusky et al. (2014) analizan el rol de los adultos en los procesos de empoderamiento de jóvenes y coinciden en el papel secundario y flexible que han de tener, aunque les atribuyen pautas claras de estímulo, apoyo, escucha y revisión. 


\section{Reflexiones finales}

Queremos acabar el artículo con unos breves apuntes sobre un par de cuestiones que merecen ser pensadas en torno a ciertas formas posibles de concebir el empoderamiento. Introduciremos estos apuntes con sendas citas literarias: unas estrofas de un poema de José Agustín Goytisolo (1994) y un aforismo de Franz Kafka (1983). Cada una de estas cuestiones podría dar lugar a desarrollos y polémicas mucho más largas; nos limitaremos únicamente a esbozarlas. Además, las citas ya son de por sí muy elocuentes.

“La vida es lucha despiadada
nadie te ayuda así nomás
y si tú solo no adelantas
te irán dejando atrás atrás.

Anda muchacho dale duro la tierra toda el sol y el mar son para aquellos que han sabido sentarse sobre los demás".

(Goytisolo, 1994: 45-46)

Probablemente muchos conocen estos versos del menor de los Goytisolo, y sobre todo el estribillo de la canción en la que los convirtió Paco lbáñez: "Me lo decía mi abuelito, / me lo decía mi papá, / me lo dijeron muchas veces/ y lo he olvidado siempre más". Papá y el abuelito advertían que siempre hay que procurar adelantar a los demás, porque sabían que las cosas (la tierra, el sol y el mar) son de quienes han aprendido a sentarse sobre los demás. Seguramente no conocían la palabra empoderamiento, pero eso que le recomendaban al muchacho también podría ser una llamada a empoderarse, una manera de adquirir poder y usarlo. Parece claro que quienes actualmente manejamos el concepto de empoderamiento no lo hacemos en el sentido de la recomendación del abuelito. Desde luego, cuando se dice que hay que empoderar a los jóvenes, no se está pensando en que a los jóvenes hay que enseñarles a sentarse sobre los demás.

No obstante, ahí se plantea una cuestión sobre la que habría que reflexionar: ¿con el poder como contenido u objetivo de la educación ocurre lo mismo que con otros contenidos u objetivos educativos? Pongamos por caso, con la cultura, en cualquiera de sus significados al uso (en sentido antropológico; cultura culta o académica, etc.). La cultura, en cualquiera de estas acepciones, es en principio susceptible de transmitirse a todos. Disponiendo de los medios pertinentes, todos podrían acceder a ella sin necesidad de repartirla o dividirla. El que uno adquiera mucha cultura, no necesariamente ha de ser a costa de que otro deba contentarse con una porción muy pequeña de la misma. De hecho, extender la cultura a cuantos más mejor tiene efectos multiplicadores de la propia cultura. ¿Pero con el poder ocurre igual?, ¿es posible que todos tengan al unísono mucho poder y puedan ejercerlo simultáneamente?, ¿no es condición de que unos tengan mucho poder que otros tengan muy poco? Si uno en casa puede mandar mucho, será porque el resto de la familia puede mandar muy poco.

Es por eso que, cuando hablamos de empoderar no estamos hablando exactamente de que todos adquieran mucho poder, sino de repartir o socializar más y mejor el poder existente. $Y$ eso significa que los procesos de empoderamiento deberían empezar con (o priorizar a) aquellas personas, grupos o colectivos que disponen de menos poder. $Y$ cuando proponemos que a todos hay que educarles para el empoderamiento, quizá lo que verdaderamente queremos decir es que, respecto al poder, lo que hay que hacer es aprender a compartirlo; aprender que todos tienen derecho a sentarse, pero -contrariamente a lo que enseñaba el abuelito del poeta- nadie debe tenerlo para sentarse sobre los demás.

"El animal arranca el látigo de la mano del amo y se azota por su cuenta para convertirse en amo de sí mismo, y no sabe que eso es sólo una fantasía, nacida de un nuevo nudo de la correa del látigo propietario".

(Kafka, 1983: 24)

A un miembro de la -según expresión de Paul Ricoeur- escuela de la sospecha, fácilmente se le podría ocurrir que la moda del empoderamiento quizá sea solo una argucia más del sistema para que el personal aprenda a controlarse solito. De hecho, el tremendo aforismo de Kafka ha sido realidad en muchas ocasiones y de mil maneras distintas: piénsese en la autoflagelación como práctica de mortificación de la carne en determinadas comunidades religiosas de antaño; en el masoquismo como perversión sexual; en la austeridad y el esfuerzo como valores máximos en la ideología del puritanismo burgués; en las comidas de coco a los adeptos para que acepten, con agrado y falsa conciencia de libertad, la subordinación total al líder sectario; etc.

Bien está que el empoderamiento sirva para arrancar el látigo de la mano del amo; pero que sea para, acto seguido, destruir el látigo y todo lo que él pueda significar; y nunca para empezar uno mismo a azotarse por su cuenta, ni, por supuesto, para ponerse entonces a flagelar a los demás. 


\section{Referencias bibliográficas}

AA. VV. (2011). Las voces del 15-M. Barcelona: Los libros del Lince.

Álvarez, K., Gallego, P., \& Gandara, F. (2011). Nosotros los indignados: Las voces comprometidas del 15-M. Barcelona: Destino.

Bauman, Z. (2010). Mundo consumo: Ética del individuo en la aldea global. Barcelona: Paidós lberica.

Boluijt, B., \& de Graaf, L. (2010). Preaching empowerment, practicing participation: The use of empowerment and citizen participation in Dutch local democracies. Toulouse: EGPA.

Carandell, J. M. (1974). La protesta juvenil. Barcelona: Salvat.

Cargo, M., Grams, G. D., Ottoson, J. M., Ward, P., \& Green, L. W. (2003). Empowerment as fostering positive youth development and citizenship. American Journal of Health Behavior, 27 (1), 66-79. doi:10.5993/AJHB.27.1.s1.7.

Carlsson, U., Tayie, S., Jacquinot-Delaunay, G., \& Pérez, J. M. (Eds.) (2008). Empowerment through media education: An Intercultural Dialogue. Sweden : The International Clearinghouse on Children, Youth and Media.

Checkoway, B. (2011). What is youth participation? Children and Youth Services Review, 33 (2), 340-345. doi:10.1016/j. childyouth.2010.09.017.

Christens, B. D., \& Peterson, N.A. (2012). The role of empowerment in youth development: A study of sociopolitical control as mediator of ecological systems' influence on developmental outcomes. Journal of Youth and Adolescence, 41 (5), 623-635. doi: 10.1007/s10964-011-9724-9.

Claret, A. (2013). Tot el poder als joves!: Apoderament juvenil i democràcia avançada en temps de crisi. Barcelona: Els Llums.

Cloete, F., \& Auriacombe, C. (2013). Measuring Empowerment in the Democratic Developmental State. Journal Home, $43(2), 1-20$

Cohn-Bendit, D., Sauvageot, J., Geismar, A., \& Duteuil, J.-P. (1970). La rebelión estudiantil. México: Serie Popular Era.

Comas, D. (2001). La evaluación de programas de ocio alternativo de fin de semana. Madrid: Injuve (Instituto de la Juventud de España).

Cornwall, A. (2008). Democratising engagement: What the UK can learn from international experience. London, UK: Demos.

Figueras, M., Soler, P., Casal, J., Saura, J.R., Romaní, O., Trilla, J., \& Feixa, C. La Ciudad Indignada. En Trilla, J. (Coord.) (2011). Jóvenes y espacio público. La ciudad indignada. (pp. 193-243). Barcelona: Bellaterra.

Fortunati, L. (2014). Media between power and empowerment: Can we resolve this dilemma? The Information Society, An International Journal, 30 (3), 169-183. doi:10.1080/01972243.2014.896676.

Freire, P. (1987). La educación como práctica de la libertad. México: Siglo XXI.

Freire, P. (1997). Pedagogía del oprimido. Madrid: Siglo XXI.

Freire, P. (1997b). A la sombra de este árbol. Esplugues de Llobregat: El Roure.

Gaventa, J. (2006). Finding the spaces for change: A power analysis. IDS Bulletin, 37 (6), 23-33. doi:10.1111/j.1759-5436.2006. tboo320.x.

Glucksmann, A. R. (2008). Mayo del 68: Por la subversión permanente. Madrid: Taurus.

Goytisolo, J. A. (1994). Palabras para Julia. Barcelona: Lumen.

Kafka, F. (1983). Consideraciones acerca del pecado, el dolor, la esperanza y el camino verdadero. Barcelona: Ed. Laia.

Kaplan, S. J., Skolnik, L., \& Turnbull, A. (2009). Enhancing the empowerment of youth in foster care: Supportive services. Child Welfare, 88 (1), 133-161.

Kronenberg, D. E. M. (2007). Towards an empowerment approach. Youth Theatre Journal, 21 (1), 129-137. doi:10.1080/08 929092.2007.10012601.

Lakin, R., \& Mahoney, A. (2006). Empowering youth to change their world: Identifying key components of a community service program to promote positive development. Journal of School Psychology, 44 (6), 513-531.

Lawrencejacobson, A. R. (2006). Intergenerational community action and youth empowerment. Journal of Intergenerational Relationships, v. 4, 137-147.

Le Goff, J-P. (1998). Mai 68, l'héritage impossible : Postface inédit de l'auteur «Mai 68 n'appartient à personne». Paris : La Découverte.

Luttrell, C., Quiroz, S., Scrutton, C., \& Bird, K. (2009). Understanding and operationalising empowerment. London: Overseas Development Institute.

Masa, M. (2009). Adolescentes en Euskadi: Una aproximación desde el empoderamiento. Vitoria-Gasteiz: EMAKUNDE-Instituto Vasco de la Mujer.

Messias, D. K., Fore, E. M., McLoughlin, K., \& Parra-Medina, D. (2005). Adult roles in community-based youth empowerment programs: Implications for best practices. Fam Community Health. v. 28, n. 4 , 320-337.

Nolas, S.-M. (2014). Exploring young people's and youth workers' experiences of spaces for "youth development": Creating cultures of participation. Journal of Youth Studies, 17 (1), 26-41. doi: 10.1080/13676261.2013.793789. 
Nussbaum, M. (2012). Crear capacidades: Propuesta para el desarrollo humano. Barcelona: Paidós lbérica.

Ozer, E. J., \& Schotland, M. (2011). Psychological Empowerment Among Urban Youth: Measure Development and Relationship to Psychosocial Functioning. Health Education \& Behavior, 38 (4), 348-356. doi:10.1177/1090198110373734.

Pato, C. (2014). Biographies for Artistic and Social Intervention: A Youth-Driven Project. Arts Education Policy Review, 115 (4), 131-140. doi: 10.1080/10632913.2014.948342.

Pearrow, M. M., \& Pollack, S. (2009) Youth Empowerment in Oppressive Systems: Opportunities for School Consultants. Journal of Educational and Psychological Consultation, 19 (1), 45-60. doi:10.1080/10474410802494911.

Peterson, N. A., Peterson, C., Agre, L., Christens, B.D., \& Michael, C. (2011) Measuring youth empowerment: Validation of a sociopolitical control scale for youth in an urban community context. Journal of Community Psychology, 39 (5), 592-605.

Qiu, J. L. (2008). Working-class ICTs, migrants, and empowerment in South China. Asian Journal of Communication, 18 (4), 333-347. doi: 10.1080/01292980802344232.

Rappaport, J. (1981). In praise of paradox: A social policy of empowerment over prevention. American Journal of Community Psychology, 9 (1), 1-25.

Rappaport, J. (1987). Terms of Empowerment/Exemplars of Prevention: Towards a Theory for Community Psychology. American Journal of Community Psychology, 15 (2), 121-148.

Reflect-Action (2009). Reflect. Recuperado de http://www.reflect-action.org/es/home.

Ricaurte, K. M., Ojeda, E., Betancourth, S., \& Burbano, H. M. (2013). Empoderamiento en jóvenes en situación de desplazamiento. El caso de la Unidad de Atención y Orientación (UAO) de la Alcaldía de Pasto. CS, 11, 177-214. doi:10.18046/ recs.i11.1570.

Richez, J.-C., Labadie, F., \& De Linares, C. (2012). «Youth empowerment » dans l'espace euroméditerranéen. Paris: INJEP.

Roth, J. L. \& Brooks-Gunn, J. (2003). What exactly is a youth development program? Answers from research and practice. Applied Developmental Science, 7 (2), 94-111. doi:10.1207/S1532480XADS0702_6.

Salvadó, A., Jiménez-Morales, M., \& Sourdis, C. (2017). El Webdoc Proyecto-HEBE: estudio de caso de documental interactivo como experiencia artística-creativa de empoderamiento juvenil. Pedagogía Social. Revista Interuniversitaria, $30, X X-X X$.

Salusky, l., Larson, R. W., Griffith, A., Wu, J., Raffaelli, M., Sugimura, N., \& Guzman, M. (2014). How Adolescents Develop Responsibility: What Can Be Learned From Youth Programs. Journal of Research on Adolescence, 24 (3), 417-430. doi: $10.1111 /$ jora.12118

San Saturnino, N., \& Gaicoechea, J. J. (2013). Liderazgo transformacional, empoderamiento y aprendizaje: un estudio en Ciclos Formativos de Grado Superior. Revista de Educación, 362, 594-622. doi:10.4438/1988-592X-RE-2013-362-243

Sen, A. (2000). Desarrollo y libertad. Barcelona: Planeta.

Trilla, J., Casal, J., Feixa, C., Figueras, M., Planas, A., Romaní, O., Saura, J., y Soler, P. (Eds) (2011). Jóvenes y espacio público. Del estigma a la indignación. Barcelona: Edicions Bellaterra.

Shaw, A., Brady, B., McGrath, B., Brennan, M. A., \& Dolan, P. (2014). Understanding youth civic engagement: debates, discourses, and lessons from practice. Community Development, 45 (4), 300-316.

Shirazi, R. (2011). When projects of "empowerment" don't liberate: Locating agency in a "postcolonial" peace education. Journal of Peace Education, 8 (3), 277-294. doi:10.1080/17400201.2011.621370.

Somerville, P. (1998). Empowerment through Residence. Housing Studies, 13 (2), 233-257. doi:10.1080/02673039883425.

Subirats, J., \& Parés, M. (2014). Cambios sociales y estructuras de poder ¿Nuevas ciudades, nueva ciudadanía? Interdisciplina, 2 (2), 97-118.

Úcar, X., Jiménez-Morales, M., Soler Masó, P., Trilla, J. (2016). Exploring the conceptualitzation and research of empowerment in the field of youth. International Journal of Adolescence and Youth. http://dx.doi.org/10.1080/02673 843.2016.1209120.

Wang, C. C. (2006). Youth participation in photovoice as a strategy for community change. Journal of Community Practice, 14 (1-2), 147-161. doi:10.1300/J125V14nO1_09.

Wilson, N., Dasho, S., Martin, a. C., Wallerstein, N., Wang, C. C., \& Minkler, M. (2007). Engaging Young Adolescents in Social Action Through Photovoice: The Youth Empowerment Strategies (YES!) Project. The Journal of Early Adolescence, 27 (2), 241-261. doi: 10.1177/0272431606294834.

Wilson, N., Minkler, M., Dasho, S., Wallerstein, N., \& Martin, A. C. (2008). Getting to social action: The Youth Empowerment Strategies (YES!) Project. Health Promotion Practice, 9 (4), 395-403. doi: 10.1177/1524839906289072.

Wong, N. (2008). A participatory youth empowerment model and qualitative analysis of student voices on power and violence prevention. Michigan: University of Michigan.

Wright, K. (2010). "Giving voice" to youths in out-of-home placements. Journal of Human Behavior in the Social Environment, 20 (3), 335-348. doi:10.1080/10911350903323844.

Zimmerman, M. (2000). "Empowerment theory". In Handbook of community psychology edited by Rappaport, J. \& Seidman, E. (Ed). New York: Kluwer Academic/Plenum Publishers. 


\section{Notas}

1 Proyecto HEBE. El empoderamiento de los jóvenes: análisis de los momentos, espacios y procesos que contribuyen al empoderamiento juvenil. Proyecto financiado per el MINECO - Programa Estatal de I+D+| Retos de la Sociedad 2013. Ref.: EDU2013-42979-R. El equipo de investigación está compuesto por P. Soler (IP); Í. Agud; J. Font; P. Heras; M. Jiménez; A. Llena; M. Monseny; A. Novella; H. Núñez; S. Páez; P. Pineda; A. Planas; P. Rodrigo; A. Salvadó; J. Trilla; N. Turon y X. Úcar. Cuenta también con la colaboración de: A. Alonso; A. Ciraso; F. Fusté y P. Garriga.

Se ha pasado P. Rodrigo como miembro del equipo investigador y no como colaboradora.

2 En él se tuvieron en cuenta 3.262 referencias bibliográficas, posteriores al año 2000, que contienen el término “Empoderamiento Juvenil” o “Indicadores de Empoderamiento". El trabajo en red de toda esta documentación se realizó a través del gestor bibliográfico Mendeley. Todo este material fue revisado por el equipo de investigación, con el criterio de escoger aquellos documentos que abordaran, de manera directa, el tema del empoderamiento juvenil desde una perspectiva socioeducativa. Se descartaron las aportaciones relacionadas con el ámbito de la salud, la medicina, la economía u otras perspectivas alejadas de nuestro interés. De este modo finalmente se seleccionaron un total de 297 referencias bibliográficas. Todos los documentos fueron etiquetados con diferentes palabras clave a partir de los objetivos de la investigación. El detalle de las etiquetas, bases de datos y criterios de análisis pueden consultarse en el artículo referenciado.

3 Marta Nussbaum (2012, pp. 400 y ss.) utiliza la expresión "capacidades internas" con un significado muy semejante al que aquí atribuimos a esta expresión. También sería interesante revisitar al maestro de Nussbaum, Amartya Sen (2000), quien también se ha referido extensamente al tema de las capacidades.

4 Nussbaum, en el libro antes citado, explica y ejemplifica muy bien esta relación mediante su concepto de "capacidades combinadas".

5 Cuando el concepto de empoderamiento se quiere aplicar no a un sujeto individual sino a la ciudadanía en general o a un colectivo determinado (los jóvenes, la infancia, las personas con necesidades educativas especiales, inmigrantes...), no hay más que substituir la palabra "persona" por la que corresponda en cada caso.

- Sobre el mayo parisino de 1968: Carandell, 1974; Cohn-Bendit, 1970 Glucksmann, 2008; Le Gofff, 1998. Y sobre el movimiento del 15 M: Álvarez, Gallego \& Gandara, 2011; Figueras et al., 2011.

7 Wilson et al. (2007) y Wilson et al. (2008) proponen las preguntas que corresponde a cada una de las iniciales en inglés del término "SHOWed": 1) ¿Qué ves aquí?, 2) ¿Qué está pasando realmente?, 3) ¿Cómo se relaciona esto con nuestras vidas?, 4) ¿Por qué existe este problema o situación?, 5) ¿Qué podemos hacer?

8 Información disponible en (http://www.reflect-action.org/es/home Consulta 25 de enero de 2017.

\section{CÓMO CITAR ESTE ARTÍCULO}

Soler, P., Trilla, J., Jiménez-Morales, M., \& Úcar, X. (2017). La construcción de un modelo pedagógico del empoderamiento juvenil: espacios, momentos y procesos. Pedagogía Social. Revista Interuniversitaria, 30, 19-34. DOI:10.7179/PSRI_2017.30.02.

\section{DIRECCIÓN COMPLETA DE LOS AUTORES}

Pere Soler Masó: Universitat de Girona. Departament de Pedagogia. Plaça de Sant Domènec, 9. 17004 Girona (Spain). pere.soler@udg.edu

Jaume Trilla Bernet: jtrilla@ub.edu

Manel Jiménez-Morales: manel.jimenez@upf.edu

Xavier Úcar Martínez: xavier.ucar@uab.edu 
PERFIL ACADÉMICO

Pere Soler Masó: Profesor titular de universidad en el Departamento de Pedagogía en la Universidad de Girona. Su actividad docente y de investigación se centra en las políticas de juventud, el asociacionismo y la participación infantil y juvenil, el desarrollo comunitario, la educación en el tiempo libre y la animación sociocultural. Actualmente es miembro del Grupo de Investigación en Políticas, Programas y Servicios Educativos y Socioculturales (GRES) del Instituto de Investigación Educativa - UdG y del Grupo de Investigación Consolidado (SGR) en Educación, participación e inclusión (GREPI) (2014SGR 774-GRC). Es coordinador general desde el 2007 del Máster Oficial Interuniversitario en Juventud y Sociedad (MIJS) impartido por seis universidades públicas. Pueden consultarse algunas de sus publicaciones en el siguiente enlace: http://girona. academia.edu/PereSolerMasó.

Jaume Trilla Bernet: Profesor universitario desde 1976 y actualmente catedrático de la Facultad de Educación de la Universidad de Barcelona. Antes había ejercido también como maestro de primaria y como educador en actividades de animación sociocultural y de tiempo libre. Autor o coautor de numerosos artículos, libros y trabajos de investigación sobre educación no formal e informal, animación sociocultural y pedagogía del ocio, desarrollo moral y educación en valores. Por algunas de estas contribuciones le han sido concedidos los Premios de Pedagogía Josep Pallach, Rosa Sensat (en dos ocasiones), Joan Profitós y Serra i Moret, así como el Premio Extraordinario de Doctorado y seis sexenios de investigación.

Manel Jiménez-Morales: Licenciado en Comunicación Audiovisual por la Universitat Pompeu Fabra y en Teoría de la Literatura y Literatura Comparada por la Universitat de Barcelona. Doctorado en Comunicación Social por la Universitat Pompeu Fabra. Ha sido guionista, productor y realizador de varios proyectos cinematográficos y televisivos, así como de programas culturales (Via Digital, Ona Catalana, Ràdio Barcelona/La SER). Contribuye, desde el ensayo y la crítica, en varias publicaciones y editoriales. Ha contribuido en varios estudios a un nivel individual y dentro de los grupos de investigación en comunicación UNICA y CINEMA. Cuenta con tres estancias de investigación (University of Oxford, University of California Los Angeles i British Film Institute) y ha impartido docencia en diversas universidades alemanas.

Xavier Úcar Martínez: Catedrático de Pedagogía Social en Dpto. de Pedagogía Sistemática y Social de la Universidad Autónoma de Barcelona. Presidente de la Sociedad Iberoamericana de Pedagogía Social (SIPS) desde el 2012. Autor de más de un centenar de trabajos que incluyen libros, capítulos de libros y artículos en revistas científicas. La última publicación (2016) es una trilogía denominada "Pedagogías de lo social", que da título al primer libro y es complementada por "Relaciones socioeducativas. La acción de los profesionales" y “Pedagogía de la elección”. Las tres editadas por "UOC Publishing" de Barcelona. http://uab.academia.edu/XavierUcar. 
